\title{
An optical NMR spectrometer for Larmor-beat detection and high-resolution POWER NMR
}

\author{
J. G. Kempf, ${ }^{\text {a) }}$ J. A. Marohn, P. J. Carson, D. A. Shykind, J. Y. Hwang, \\ M. A. Miller, and D. P. Weitekamp \\ A. A. Noyes Laboratory of Chemical Physics, M/S 127-72, California Institute of Technology, \\ Pasadena, California 91125, USA
}

(Received 22 March 2008; accepted 3 May 2008; published online 11 June 2008)

\begin{abstract}
Optical nuclear magnetic resonance (ONMR) is a powerful probe of electronic properties in III-V semiconductors. Larmor-beat detection (LBD) is a sensitivity optimized, time-domain NMR version of optical detection based on the Hanle effect. Combining LBD ONMR with the line-narrowing method of POWER (perturbations observed with enhanced resolution) NMR further enables atomically detailed views of local electronic features in III-Vs. POWER NMR spectra display the distribution of resonance shifts or line splittings introduced by a perturbation, such as optical excitation or application of an electric field, that is synchronized with a NMR multiple-pulse time-suspension sequence. Meanwhile, ONMR provides the requisite sensitivity and spatial selectivity to isolate local signals within macroscopic samples. Optical NMR, LBD, and the POWER method each introduce unique demands on instrumentation. Here, we detail the design and implementation of our system, including cryogenic, optical, and radio-frequency components. The result is a flexible, low-cost system with important applications in semiconductor electronics and spin physics. We also demonstrate the performance of our systems with high-resolution ONMR spectra of an epitaxial AlGaAs/GaAs heterojunction. NMR linewidths down to $4.1 \mathrm{~Hz}$ full width at half maximum were obtained, a $10^{3}$-fold resolution enhancement relative any previous optically detected NMR experiment. (c) 2008 American Institute of Physics. [DOI: 10.1063/1.2936257]
\end{abstract}

\section{INTRODUCTION}

Optical nuclear magnetic resonance (ONMR) is an extremely high-sensitivity probe of Group III-V semiconductors. ${ }^{1-9}$ It has been implemented with state-ofthe-art NMR pulse sequences, ${ }^{4-6,8,9}$ and applied to samples down to single quantum dots of just $10^{5}$ nuclei. $^{7}$ ONMR is an informative probe of quantum-confined electronic systems, including those features that control their behavior in device applications. Exemplary results have detailed single-carrier distributions of electron spin and charge, ${ }^{9}$ local electric fields that give rise to band bending at material interfaces, ${ }^{9}$ fewelectron cooperative behavior that minimizes spin and Coulombic energies in a two-dimensional electron gas, ${ }^{10}$ and lateral distributions of strain in GaAs quantum wells. ${ }^{11}$

The key to ONMR is the coupling of photoexcited electrons to lattice nuclei via the contact hyperfine interaction. In III-V materials, circularly polarized light excites spinpolarized electrons whose relaxation induces fluctuations in the contact hyperfine interaction, thus transferring spin order to lattice nuclei. ${ }^{1,2}$ This optical nuclear polarization (ONP) process enhances NMR sensitivity more than three orders of magnitude. ${ }^{6}$ Further dramatic sensitivity enhancements may be obtained by using the same electron-nuclear coupling for optical detection of the NMR signal. Various methods have been developed to encode NMR signals in the polarization of

\footnotetext{
${ }^{a}$ Present address: Department of Chemistry and Chemical Biology, Rensse-
} laer Polytechnic Institute, Troy, NY 12180. photoluminescence (PL), ${ }^{2-4,6,12,13}$ in optical frequency shifts ${ }^{7}$ or in Faraday rotation of a linearly polarized optical probe. ${ }^{14}$ Here, we detail implementation and advantages of the PL-based Larmor-beat-detection (LBD) method. ${ }^{6}$

Achieving state-of-the-art solid-state NMR resolution in an ONMR experiment requires time-domain nuclear spin evolution and sophisticated radio-frequency (rf) pulse sequences that coherently manipulate the NMR Hamiltonian. Buratto et al. ${ }^{4}$ introduced a time-domain ONMR paradigm in which ONP, NMR evolution, and optical detection are sequentially executed. This time-sequenced approach avoids perturbation of the NMR evolution period with the photoexcited electrons required for optical detection. As first implemented, time-sequenced ONMR encoded the NMR signal as a steady state Hanle-effect depolarization of PL. Subsequently, Marohn et al. ${ }^{6}$ provided the LBD method, a sensitivity optimized version of Hanle-effect optical detection that also enables real-time detection of the precessing transverse nuclear magnetization. More recently, time-sequenced ONMR with LBD was used to image distributions of spin and electric field corresponding to electronic states in an epitaxial AlGaAs/GaAs heterostructure. ${ }^{9}$ These experiments utilized a novel method for characterizing small, cyclic perturbations to the sample without interference from order-ofmagnitude larger static spin interactions, an approach we refer to as power (perturbations observed with enhanced resolution) NMR. ${ }^{5,8,9}$

To date, few detailed descriptions of ONMR instrumentation and procedures have been presented. ${ }^{13,15}$ The goal of 
the present paper is to present the features of our apparatus that enable the unique combination of optical NMR with both LBD and POWER NMR imaging. Many aspects are equally relevant to alternate versions of optically detected NMR. ${ }^{13,14}$ Features of our apparatus include (a) multinuclear rf excitation, (b) systems to cycle the magnitude and orientation of the static magnetic field $\left(\mathbf{B}_{0}\right)$, (c) controls to synchronize optical pulses with NMR pulse sequences, and (d) operation with the sample in superfluid He. Multinuclear excitation is invaluable for probing the isotopic dependence of observables from quadrupole splittings to spin relaxation rates. Furthermore, LBD requires generation of simultaneous transverse magnetization on two isotopes. In GaAs, the III-V material most commonly studied by ONMR, there are three spin species, ${ }^{69} \mathrm{Ga}(60 \%),{ }^{71} \mathrm{Ga}(40 \%)$, and ${ }^{75} \mathrm{As}(100 \%)$. Our apparatus allows three-isotope excitation with an exceptionally simple, untuned, single-coil design, and digital rf synthesis. The second noted feature, variable $\mathbf{B}_{0}$, is used to set the orientation of NMR quantization axes with respect to the sample crystal axes. This degree of freedom allows exploration of orientation-dependent spin Hamiltonians, such as that for the quadrupole interaction. Finally, the noted capability for synchronization enables high-resolution measurement of optically induced sample perturbations with POWER NMR. We have used this method to image the distributions of spin density and electric field from single-electronic states, ${ }^{9}$ and anticipate broader applications to probe electronic features relevant to samples of interest in fields from quantumconfined solid-state physics ${ }^{16-18}$ to spintronics applications. ${ }^{19}$

\section{CRYOGENIC SYSTEMS}

ONP and optical detection require cryogenic sample temperatures to ensure that fluctuations of the contact hyperfine interaction dominate nuclear spin relaxation. We use a $l$-He immersion, optical cryostat (Pope Scientific) operated below the $\lambda$-point $(T \sim 2 \mathrm{~K})$ of liquid helium. This avoids bubbling that would interfere with optical aspects, while the high thermal conductivity of superfluid helium ${ }^{20}$ enhances tolerance to power deposited by NMR pulse sequences. Nonetheless, operation in $\mathrm{He}$ gas and even with a $l-\mathrm{N}_{2}$ optical cryostat $^{21}$ at $77 \mathrm{~K}$ is feasible for some ONMR experiments. $^{2}$

A cross-sectional cryostat schematic is shown in Fig. 1. Internal glass walls separate vacuum and $l-\mathrm{N}_{2}$ insulation jackets from the $101 l$-He immersion chamber. The vacuum jacket is prepared at $10^{-5}$ torr via stopcock access (not shown). A 3.0 in. diameter copper flange at top accommodates the sample probe and magnet assembly (see Fig. 2), while a 2.0 in. diameter sidearm connects to a $1.5 \mathrm{in.}$ $\times 30 \mathrm{ft}$ copper pipe to the mechanical pump (Duo Seal, model 1397). The latter pumps at $\sim 1801 / \mathrm{min}$ to adiabatically cool $l$-He from $4.2 \mathrm{~K}$ to the $\lambda$-point in $40 \mathrm{~min}$ with $\sim 40 \%$ loss. The $l$-He hold time at the $\lambda$-point ranges from 5 to $7.5 \mathrm{~h}$ depending on the rf power demands and corresponding heat input of particular experiments.

Optical access is via 1.25 in. diameter external quartz windows, followed by $1.5 \mathrm{in}$. of vacuum space, a second quartz window of 0.75 in. diameter and, finally, $\sim 1.5$ in. of

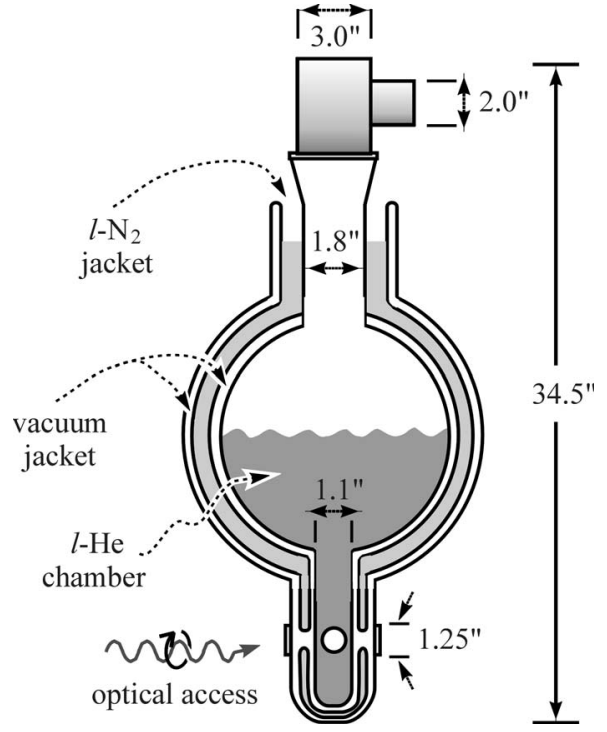

FIG. 1. Cross-sectional schematic of the $l$-He optical cryostat, custom made by Pope Scientific from $0.23 \mathrm{~cm}$ thick borosilicate glass.

$l$-He to the sample. The external windows are attached to the cryostat by using waxlike Dekhotinsky cement (Pope Scientific). Three optical windows are included. Only one is used for excitation and detection, though an orthogonally facing window provides a convenient view of the sample.

\section{NMR PROBE AND MAGNET ASSEMBLY}

A probe schematic is shown in Fig. 2. It includes the primary static field source, rf excitation coils, a sample mount, and temperature monitoring via a carbon glass resistor (Lakeshore Cryogenics). The probe head consists of a $7.2 \mathrm{~cm}$ diameter, $3.4 \mathrm{~cm}$ thick brass cap that mates the vertical copper flange of the cryostat with an O-ring sealed compression fitting. A standard $l$-He transfer line connects to an

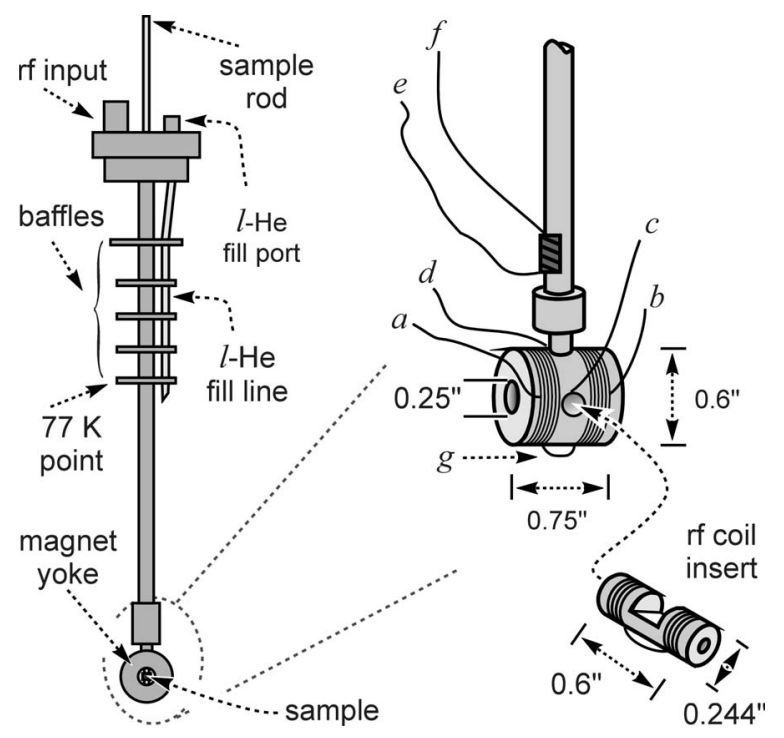

FIG. 2. ONMR probe design. The inset depicts the magnet assembly and $\mathrm{rf}$ coil insert. Electrical leads to the magnet are at $a$ and $b$, while the pair is joined at $g$. The rf insert, with leads $c$ and $d$, fits snugly in the magnet assembly and is shown in more detail in Fig. 3. Leads to the temperature sensor are at $e$ and $f$. Pressure release (5 psi) at the probe head is not shown. 
O-ring sealed fitting at the probe head, which is followed by an aluminum pipe for delivery to the main chamber. Excepting electrical fittings, the remainder of the probe is constructed from the glass-epoxy laminate, G-10, including circular baffles that block heat transfer to the $77 \mathrm{~K}$ cryostat neck. G-10 parts were cemented by using a thermalexpansion-matched epoxy of equal parts resin (CIBA 6010), hardener (CIBA 825), and fine quartz chop cured in air for $8 \mathrm{~h}$.

Superconducting magnets are a convenient, low-power source of magnetic field. Our probe incorporates a homewound superconducting Helmholtz pair (Fig. 2) for the socalled longitudinal $\mathbf{B}_{0}$, which is roughly parallel to the axes of optical excitation and detection. This simple design was sufficient because our experiments require field homogeneity only over an effective sample volume set by the optical spot size (here, $\sim 350 \mu \mathrm{m}$ ) and the micron-scale absorption depth. The magnet is wound on a G-10 form with 54filament niobium-titanium alloy wire. Leads are No. 13 copper wire for the top 6 in. (above probe baffles), with the remainder of $\mathrm{Nb}-\mathrm{Ti}$ with multiple solder connections to a parallel No. 16 brass wire, which serves as the main conductor in portions exposed upon $l$-He boiloff. Thus, operation is nonpersistent, but dissipation is low and confined to the gas phase above the superfluid $l$-He. Detailed rationale for the selected wiring materials is given elsewhere. ${ }^{22}$ The magnet inductance is $1.67 \mathrm{mH}$ at $2 \mathrm{~K}$, while field strength is $20.5 \mathrm{mT} / \mathrm{A}$. A G-10 plug mounts a rf coil insert that fits into the magnet assembly and connects to a No. 24 stainless steel coaxial cable that runs to the probe head. A notch in the insert accommodates the tip of a G-10 rod to which we affix one or more samples for delivery down the hollow center of the probe. Typically, we operate at a field of $B_{0} \sim 250 \mathrm{mT}$, as calibrated by NMR. This value balances sensitivity of LBD detection against the linearity of its response function. ${ }^{6,23}$ At the optimal position of the Hanle curve, the slope of this function with respect to the measured nuclear hyperfine fields is inversely proportional to the total field experienced by luminescent electrons. Too small, a value of $B_{0}$ would reduce the total field to the point where the response would be highly nonlinear.

The rf insert is detailed in Fig. 3(a). It enables multinuclear excitation of all isotopes in GaAs [Fig. 3(b)] and can easily be made to cover other III-V isotopes. This usability with a single untuned and unmatched coil is a side benefit of LBD, which is well suited to fields $<1 \mathrm{~T}$. Such low fields advantageously limit the frequency range needed to cover multinuclear excitation. All frequencies were digitally synthesized and amplified (ENI, model 500A, 0.3-35 MHz, $500 \mathrm{~W}$ amplifier) in a single channel. Higher $B_{0}$ would require a complicated, multiply tuned excitation circuit and would not yield any advantage, as sensitivity is determined by ONP and optical detection.

\section{EVENT CONTROL AND SYNCHRONIZATION}

Primary control of our time-sequenced, LBD-detected ONMR experiments is from a C-language program run on a standard personal computer (PC). For each experiment, the

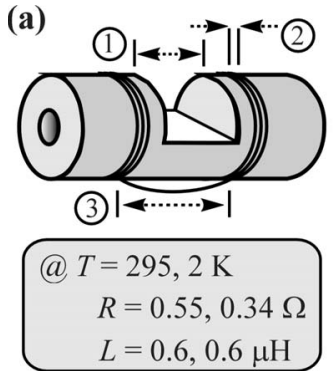

(b)

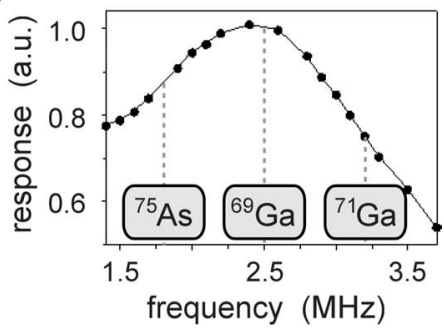

FIG. 3. (a) Schematic of rf coil insert. The coil is a joined pair of $36 \mathrm{AWG}$ insulated copper wire, with closed-packed halves in three levels of three windings each. The notch (1) for sample-rod access is 0.125 in. wide, while the inner edges (2) of the $0.020 \mathrm{in}$. deep by $0.021 \mathrm{in}$. wide coil-winding grooves are separated (3) by 0.175 in. A 0.185 in. diameter hole on the long axis allows sample viewing. Electrical characteristics at $T=295$ and $2 \mathrm{~K}$ are noted. (b) Coil response to direct amplifier input, with GaAs Larmor frequencies at $B_{0}=250 \mathrm{mT}$ indicated. At the ${ }^{71} \mathrm{Ga}$ resonance, we have obtained $B_{1} \sim 6.9 \mathrm{mT}$, for a $90 \mathrm{kHz}$ Rabi frequency, using $\sim 450 \mathrm{mVp}$.p. input to the ENI-500A amplifier.

program defines three parallel instruction sets. As shown in Fig. 4, one is passed to an external digital word generator (Interface Technologies, model RS-670) via a generalpurpose interface bus (GPIB) (National Instruments, model AT-GPIB) in a PC expansion slot. The digital word generator provides up to 16 channels of loopable transistor-totransistor logic (TTL) instructions on a $40 \mathrm{MHz}$ clock (25 ns resolution) that is provided externally by a precision source (Programmed Test Sources, model PTS-500). A second instruction set is loaded to a two-channel, 12-bit arbitrary waveform generator (Signatec, model AWG502) in a PC expansion slot. The AWG502 digitally synthesizes and times the output sequences of rf pulses with arbitrary phase, amplitude, and frequency. It is synchronized with TTLcontrolled events by sourcing the AWG502 clock from the digital word generator and initiating the rf timeline with a TTL trigger. Empirically, the AWG502 initiates 700 ns after the rising edge of the TTL trigger. Thus, we use a trigger of that duration to align the two timelines. Finally, the third parallel instruction set is executed by the $\mathrm{C}$ program itself via GPIB communication to additional peripherals. Here,

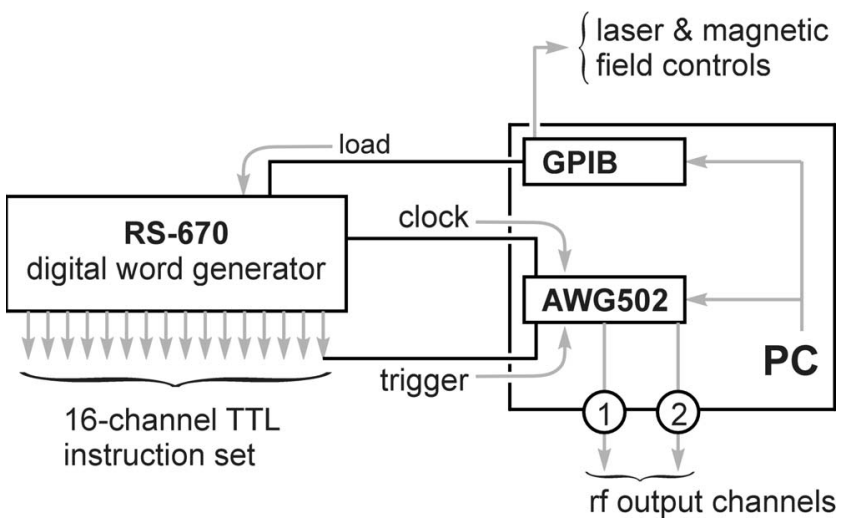

FIG. 4. Schematic of automated control elements in the LBD ONMR experiment. Four interconnected instruments determine event timing: a personal computer (PC) with general-purpose interface bus (GPIB) and arbitrary rf waveform generator (AWG502) plug-in boards, and an external digital word generator (RS-670). 


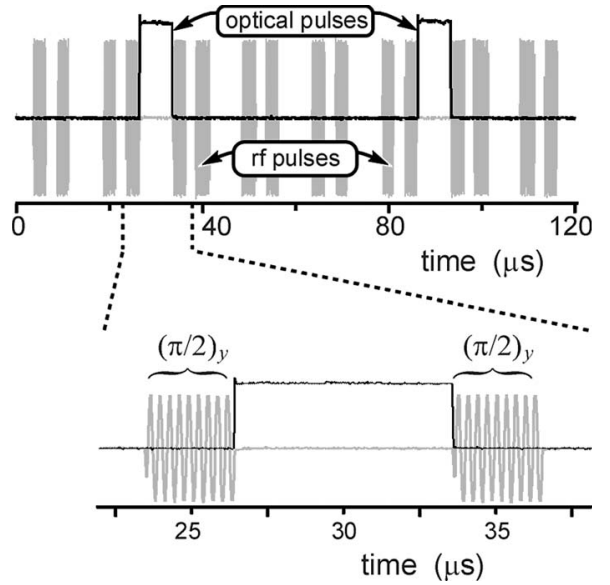

FIG. 5. Synchronized rf and optical events in a POWER NMR experiment. The $120 \mu$ s segment shown was preceded by $2.0 \mathrm{~s}$ parallel operation of the AWG502 (rf pulses) and RS-670 (TTL-controlled optical pulses). This ensured sensitivity to any accrued timing error. The expanded view highlights the arrival of an optical pulse between two $\pi / 2 \mathrm{rf}$ pulses with accuracy to $\leqslant 100 \mathrm{~ns}$.

timing relies on the independent PC clock and is only roughly synchronized. Software polls to the PC system timer via the ftime ( ) $\mathrm{C} / \mathrm{C}++$ function were updated at $18.2 \mathrm{~Hz}$, resulting in worst-case jitter of $60 \mathrm{~ms}$. This was easily accommodated by placing GPIB-controlled events within sufficient delays on the TTL and rf timelines.

POWER NMR experiments require synchronization of $\mathrm{rf}$ and TTL-controlled events to $\leqslant 100$ ns over a time line extending several seconds. ${ }^{5,8,9}$ Here, the measured perturbation is due to optical excitation. Successful rf/optical coordination is demonstrated in Fig. 5, which plots simultaneously digitized rf from the AWG502 and TTL-controlled laser output monitored with a photodiode. The sequence shown combines optical excitation (7.6 $\mu$ s pulses of $\mathrm{cw}$ irradiation) in windows of the CLSW-16 multiple-pulse line-narrowing sequence, ${ }^{24}$ here composed of $\sim 3.45 \mu \mathrm{s} \pi / 2$ rf pulses. The optical pulses provide spin-polarized electrons that induce a distribution of contact hyperfine (Knight) shifts over nuclei within the optically excited state. This cyclic perturbation provides an observable average Hamiltonian for the hyperfine interaction that dominates coherent evolution while static interactions are removed by CLSW-16. ${ }^{5,8,9}$ The demonstrated synchronization of perturbation and pulse sequence to a small fraction of the $25 \mathrm{~ns}$ clock period ensured accurate knowledge of the magnitude and operator form of the resulting average Hamiltonian.

\section{OPTICAL SYSTEMS}

The optical excitation pathway is depicted in Fig. 6(a). The collimated, linearly polarized output $(32 \mathrm{~mW}$ maximum) of an $802.1 \mathrm{~nm}$ diode laser (Melles Griot, model 06DLL707) passes through a focusing lens (focal length, $f=40 \mathrm{~cm}$ ), followed by a $\lambda / 4$ plate that converts the light to right $\left(\sigma_{+}\right)$circular polarization $(\mathrm{CP})$. After passage through cryostat windows, the excitation beam reaches the sample at a distance of $\sim 45 \mathrm{~cm}$ from the focusing lens, yielding a measured spot diameter of $\sim 350 \pm 50 \mu \mathrm{m}$. The beam propagates $\sim 15^{\circ}$ off axis of $\mathbf{B}_{0}$ and the surface normal of the
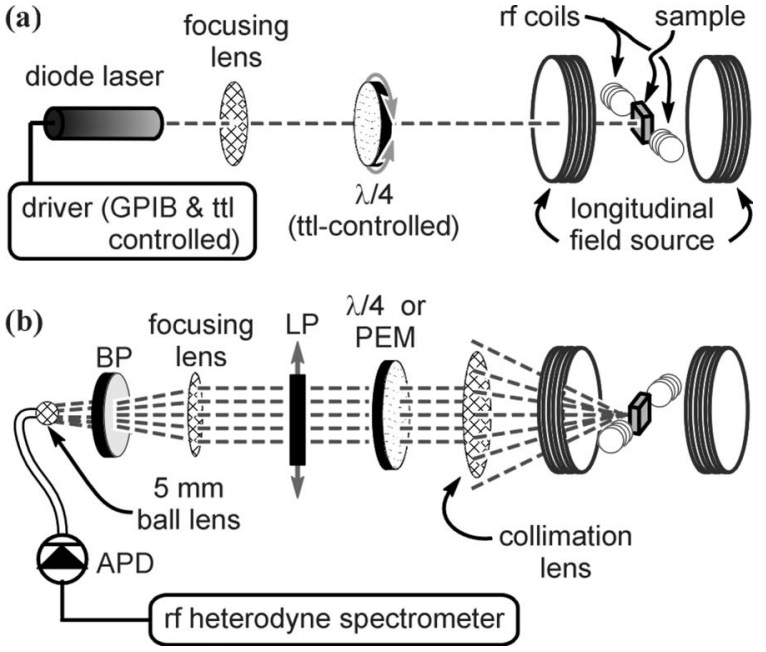

FIG. 6. Pathways of (a) optical excitation and (b) detection. In the detection arm, BP is an optical bandpass filter, LP is a linear polarizer, and APD is a fiber-coupled avalanche photodiode. A quarterwave plate $(\lambda / 4)$ or photoelastic modulator (PEM) is used for polarization analysis in the distinct configurations discussed in the main text.

sample. This off-angle excitation allows the detection pathway to be parallel to $\mathbf{B}_{0}$, while slightly reducing the photoexcited electron-spin polarization. ${ }^{2}$

Automated control is required to separately optimize excitation during NMR evolution, ONP, and detection periods. A diode laser driver (Melles Griot, model 06DLD103) enables TTL control of laser high and low power levels (as well as on/off). High/low settings and other features are accessible via the GPIB. Finally, a TTL-controlled stepper motor (Superior Electronics, model MO 61-FD-6244) and driver (Forthright Electronics, model STPNA2) enable automated rotation of a geared, homebuilt optics holder for the $\lambda / 4$ plate. For example, rotation by $90^{\circ}$ switches from $\sigma_{+}$to $\sigma_{-}$ excitation in 50 steps over $300 \mathrm{~ms}$.

The optical detection pathway is parallel to $\mathbf{B}_{0}$ and the sample surface normal ([001] crystal axis). This is optimal for correlation of PL polarization with electron-spin polarization. Two arrangements of the detection optics are used: one for pre-NMR "tuning" of the optics to maximize polarized PL intensity and a modification on that setup appropriate for LBD.

\section{A. The tune-up configuration}

The apparatus for this arrangement is shown in Fig. 6(b). The sample in place for this procedure is typically the $\mathrm{Al}_{x} \mathrm{Ga}_{(1-x)} \mathrm{As} / \mathrm{GaAs} \quad(x=0.36)$ heterojunction sample described ahead in Sec. VIII. During CP excitation, PL passes out of the cryostat through two consecutive optical windows and to a lens $(f=10 \mathrm{~cm})$, located $\sim 5 \mathrm{~cm}$ from the outer window, that nominally collimates the PL. An approximately $3 \times 3 \mathrm{~mm}^{2}$ notch is cut into the outer edge of this lens to prevent interference with the excitation beam. The PL then passes through a photoelastic modulator (PEM) (Hinds International, model 0101) driven at $50 \mathrm{kHz}$ between the $\pm \lambda / 4$ conditions of the wavelength $\lambda$ that carries the ONMR signal. A linear polarizer (LP) follows, then a focusing lens $(f=10 \mathrm{~cm})$ spaced $\sim 52 \mathrm{~cm}$ from the collimation lens. An 
optical bandpass (BP) filter permits transmission of desired luminescence components to a $5 \mathrm{~mm}$ spherical ball lens that is mounted $\sim 8.5 \mathrm{~cm}$ from the focusing lens. The BP filter contributes to ONMR sensitivity by limiting contributions to photon shot noise and to its selectivity when the PL contains more than one potential NMR signal carrier. The ball lens couples the polarization-analyzed light into the free end of an optical fiber $(200 \mu \mathrm{m}$ core, 0.24 NA, PC-polished, multimode) that is permanently coupled to an avalanche photodiode (APD) (EG\&G Optoelectronics, model C30657-010QC-06).

The PEM-LP combination provides polarization analysis for optimization of the PL signal. In the first half cycle of PEM modulation, $\sigma_{+}$and $\sigma_{-}$components are transformed into orthogonal linearly polarized waves, e.g., $x$ and $y$. The LP passes only one of these. In the second-half cycle, the conversion reverses to pass the other component. Thus, the photocurrent at the $50 \mathrm{kHz}$ PEM frequency measures the difference $\left(\sigma_{+}-\sigma_{-}\right)$in $\mathrm{CP}$ intensities, which we observe at a lock-in amplifier (Stanford Research Systems, model SR510). Simultaneous mechanical chopping of the laser excitation at $\sim 1.5 \mathrm{kHz}$ [not shown in Fig. 6(a)] provides the total PL signal $\left(\sigma_{+}+\sigma_{-}\right)$for lock-in detection. Initial alignment to optimize the total PL, is followed by fine adjustments to the positioning of optics to maximize the CP difference signal. Hanle effect ${ }^{2,12,25}$ depolarization of the signal by an applied orthogonal magnetic field confirms the origin of the difference in the sample PL (e.g., as distinct from residual polarization of scattered or reflected light). An $\sim 50 \mathrm{mT}$ transverse magnetic field from Helmholtz pair electromagnets external to the cryostat (not shown in Fig. 6, but detailed in Sec. VIII) is sufficient to reduce $\left(\sigma_{+}-\sigma_{-}\right)$to zero. Finally, orienting the longitudinal field antiparallel to the optically pumped electron spins yields greater PL polarization than the parallel case (the so-called banana effect). ${ }^{22,26-28}$ Thus, for ONMR, we bias the current source for an antiparallel field given the $\mathrm{CP}$ of the incident light.

\section{B. The configuration for LBD}

LBD signals are observed as a rf modulation of one $\mathrm{CP}$ component of PL at the difference $\nu_{\mathrm{LBD}}=\left(\nu_{0}^{\mathrm{sig}}-\nu_{0}^{\text {ref }}\right)$ between the Larmor frequencies of the NMR signal nucleus and a reference isotope. ${ }^{6}$ The resulting rf component of APD output is transmitted to a heterodyne spectrometer (Sec. VI). To detect it, we modify the "tune-up" configuration by (1) replacing the PEM with a static $\lambda / 4$ oriented relative to the LP axis to transmit $\sigma_{-}$only, and (2) removal of the chopper from the excitation pathway. Alternatively, Larmor beats may instead be observed as an additional modulation of the difference signal by retaining the PEM. ${ }^{6}$ However, that arrangement provides no advantage over LBD-only modulation and requires an additional $50 \mathrm{kHz}$ demodulation of the signal.

\section{Collection of PL spectra}

Measurement of the PL spectrum and polarization complements study of the luminescent electronic states by ONMR. We obtained PL spectra by replacing the APD with a fiber-coupled (200 $\mu \mathrm{m}$ core, multimode) optical spectrom- eter (Ocean Optics, model PC2000). Total PL spectra, or $\sigma_{+}$ or $\sigma_{-}$component spectra, were collected by excluding or including the $\lambda / 4$ plate in the detection pathway.

\section{THE rf HETERODYNE NMR SPECTROMETER}

Detection of the LBD signal in a $20 \mathrm{~Hz}$ bandwidth is accomplished with the rf receiver design of Fig. 7. A transimpedance amplifier packaged with the APD converts the photocurrent to a voltage with primary components at $\nu_{\mathrm{LBD}}$ and dc. The latter is due to incomplete modulation of PL by the nuclear signal magnetization, and is immediately filtered [segment (1), Fig. 7] as APD output passes through a combination $80 \mathrm{kHz}$ high pass filter and 10:1 impedance transformer (TTE, custom order, serial H579-80K-6220) that also matches the $50 \Omega$ input impedance of the spectrometer. A $1.5 \mathrm{MHz}$ low pass filter (homebuilt) is used to prevent spurious high-frequency APD output from saturating the lownoise preamp (Mini-Circuits, ZFL-500LN) of segment (2) in Fig. 7. One or two preamps are used in series depending on the required gain. The signal is then mixed (Mini-Circuits, model ZAD-1H $)$ with a reference wave at $\nu_{\text {ref }}=(30 \mathrm{MHz}$ $\left.-\nu_{\mathrm{LBD}}\right)$ and filtered to yield an intermediate signal carrier at $30 \mathrm{MHz}$. This reference is a single sideband obtained by mixing a wave at $\nu_{\mathrm{LBD}}$ from the AWG502 with the conditioned $30 \mathrm{MHz}$ output of the same PTS-500 that provides its clock via the RS-670 digital word generator.

Phase-sensitive NMR detection occurs in the third segment of Fig. 7. The $30 \mathrm{MHz}$ signal carrier is split into two components $90^{\circ}$ out of phase. Each is mixed with conditioned $30 \mathrm{MHz}$ PTS output whose phase is adjusted to null one dc signal component, thereby maximizing the other. Finally, each channel feeds through a $10 \mathrm{~Hz}$ low pass filter (Stanford Research Systems, model SR640) for 16 bit digitization at $20 \mathrm{~Hz}$ (Computer Boards, model CIO-DAS 1402), as triggered by a $1 \mu$ s TTL pulse from the RS-670 of Fig. 4 . A home-written $\mathrm{C}$ program provides graphical output of the sequence of digitized signal transients collected during an ONMR experiment.

\section{REORIENTATION OF THE MAGNETIC FIELD}

During ONP and LBD, the static magnetic field must be parallel, or nearly so, to the axes of optical pumping and detection. However, its unrestricted orientation during NMR evolution enables study of the angular dependence of nuclear spin interactions. Electric-field-induced ${ }^{8,29-33}$ or strain-induced ${ }^{6,34,35}$ quadrupole interactions have distinct dependence on magnetic-field orientation and are particularly relevant in our studies. ${ }^{6,8,9}$ These arise, to first order, from the distortion of noncentrosymmetric crystals. In GaAs, electricfield induced resonance splittings vanish when $\mathbf{B}_{0}$ is along the [001] growth axis ${ }^{8,31}$ (as for ONP and LBD). Thus, field cycling to an active orientation for NMR evolution is desirable. An experiment timeline accommodating this is shown in Fig. 8(a).

Our apparatus includes a secondary $\mathbf{B}_{0}$ source from an electromagnet external to the cryostat whose field is orthogonal to both the longitudinal source and the rf excitation coils. This "transverse" source is an approximate Helmholtz pair 
(1) LBD signal detection \& filtering

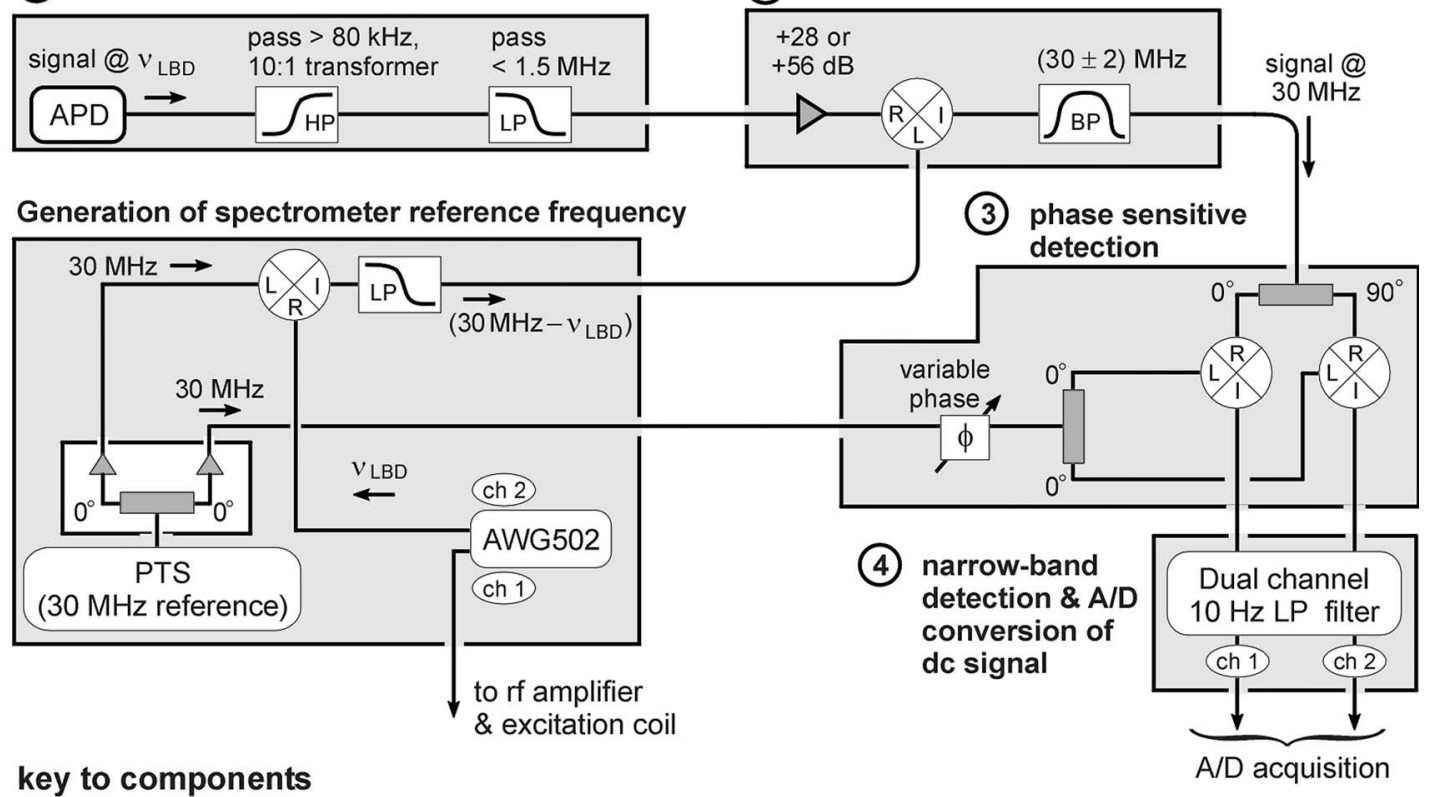

\begin{tabular}{|ll|l|}
\hline & $\begin{array}{l}\text { rf amplifier } \\
\text { high-pass filter } \\
{[\text { low- (LP) \& bandpass (BP) ] }}\end{array}$ & $\alpha$
\end{tabular}

FIG. 7. Schematic of the ONMR spectrometer. Some amplification steps have been omitted for the sake of clarity. The net spectrometer gain is 73 or $45 \mathrm{~dB}$ for high or low preamp settings.

with coils of $31 \times 31$ windings of square $(1.7 \mathrm{~mm})$ copper wire supported on a copper yoke with $31 \mathrm{~cm}(19 \mathrm{~cm})$ outer (inner) diameter and $5.7 \mathrm{~cm}$ width. The yokes are separated by $12 \mathrm{~cm}$ in an aluminum stand that may be rotated by $\pm 12^{\circ}$ for further investigation of the orientation-dependent spin interactions. Room-temperature electrical characteristics of this water-cooled pair are $R=11.0 \Omega, L=99.1 \mathrm{mH}$, and $C$ $=197 \mathrm{nF}$. It provides $5.65 \mathrm{mT} / \mathbf{A}$ at the sample and typical operation is at $57.8 \mathrm{mT}\left[\nu_{0}\left({ }^{71} \mathrm{Ga}\right)=750 \mathrm{kHz}\right]$.

Field cycling between longitudinal and transverse sources is accomplished via GPIB control of a two-channel analog voltage output that is an incidental feature of a lock-in amplifier (Stanford Research Systems, model SR510). The first channel modulates a homebuilt current limiter (Fig. 9), which, in turn, gates output of the power supply (Hewlett Packard, model 6264B) to the longitudinal magnet. The other channel controls a power supply (Hewlett Packard, model 6675A) connected directly to the transverse magnet. The control voltages range from 0 to $10 \mathrm{~V}$ with $2.5 \mathrm{mV}$ resolution, while the current-control circuitry for the longitudinal and transverse fields provides 2.24 and $2.78 \mathrm{~A} / \mathrm{V}$, respectively. These translate to resolutions of 0.12 and $0.05 \mathrm{mT}$, or, in terms of $\nu_{0}\left({ }^{71} \mathrm{Ga}\right), 1.5$ and $0.65 \mathrm{kHz}$.

As shown in Fig. 8(a), field cycling is accommodated by delays before and after NMR evolution. During these periods, spins initially aligned with $\mathbf{B}_{0}$ adiabatically follow its trajectory to a new orientation. Figure 8(b) shows digitized field cycling events starting from the longitudinal field used for ONP, after which the transverse field is cycled to half its final value in about $300 \mathrm{~ms}$. Next, the longitudinal field is dropped to zero as the transverse field is switched to its ul- timate value for NMR evolution. This two-step process prevents level crossing among nuclear Zeeman energy levels, which would lead to fast decay of the spin order obtained during ONP. The requisite time for a cycle from a longitudinal field of $246.5 \mathrm{mT}$ to a stable transverse field of $57.8 \mathrm{mT}$ is $2.1 \mathrm{~s}$, which encompasses both the obvious changes shown in Fig. 8(b) and the complete damping of small oscillations below the plotted scale, which would significantly impact NMR experiments. Following the first cycling event, a $\pi / 2$ pulse rotates spin magnetization $90^{\circ}$ form $\mathbf{B}_{0}$ as preparation for evolution during $t_{1}=\left(n \Delta t_{1}\right)$, which is terminated by a $\pi / 2$ pulse which stores one component of the precessing spin magnetization from $t_{1}$ along $\mathbf{B}_{0}$ for adiabatic reorientation to the longitudinal geometry. This allows time for the second field cycling event, which is an approximate reversal of the first two-step process and requires $700 \mathrm{~ms}$. The adiabatic condition $^{36}$ requires that a reduced cycling rate, $\left(\nu_{c} / \gamma_{n} B_{\text {tot }}\right)$ $\ll 1$, where $B_{\text {tot }}$ and $\nu_{c}=(d \theta / d t) / 360$ are the instantaneous magnitude and reorientation rate of the total field, respectively, and $\gamma_{n}$ is the gyromagnetic ratio of aligned spins. In GaAs, ${ }^{75}$ As has the smallest $\gamma_{n}$, and thus the most restrictive limitation on $\nu_{c}$. In our scheme, the reduced rate for ${ }^{75} \mathrm{As}$ easily fulfills the adiabatic criterion for both cycling events, as shown for the peak portion of the first event in Fig. 8(c).

Finally, a $\pi / 2$ pulse follows the completed second field cycling event. This pulse rotates spin magnetization from the NMR signal nucleus into the transverse plane for readout during LBD optical detection. ${ }^{6}$ The method also incorporates transverse magnetization from a "reference" nucleus that is spin-locked off resonance. The dynamic vector sum of signal 


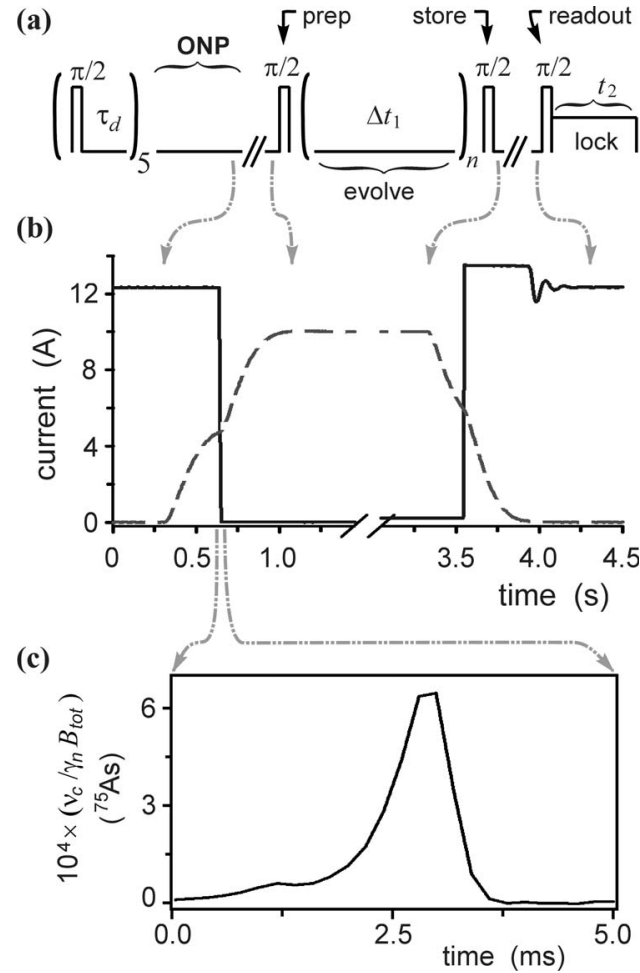

FIG. 8. (a) General ONMR rf pulse sequence that accommodates field cycling before both evolution in $t_{1}$ and LBD in $t_{2}$. Preparation, store, and readout $\pi / 2$ pulses are defined in the main text. (b) Magnet current in longitudinal (solid) and transverse (dashed) sources plotted on a timeline for the sequence in (a). The time required for the first cycling event, noted in the text, to be $\sim 2.1 \mathrm{~s}$, appears to be shorter in this plot because the vertical scale is much larger than small $(<0.1 \mathrm{~A})$ oscillations in the longitudinal source that occur to $\sim 2.0 \mathrm{~s}$ beyond its initial drop to $\sim 0 \mathrm{~A}$. Such oscillations are similar in form to those shown for the longitudinal source after it cycles up in the second cycling event. (c) ${ }^{75}$ As reduced cycling rate through its maximum during the first cycling event in (b). The reduced rate $(\ll 1)$ easily satisfies the adiabatic requirement.

and reference nuclear hyperfine fields elicits a PL polarization response in the optimally sensitive and linear region of the Hanle curve. The observed signal is a modulation of PL polarization at the difference, $\nu_{\mathrm{LBD}}$, between the Larmor frequencies of signal and reference nuclei.

\section{STRAIN-FREE SAMPLE MOUNTING AND HIGH-RESOLUTION ONMR}

Samples are affixed to the flattened end of the sample rod with a thin layer of silicone high-vacuum grease (Dow Corning). We used an $\mathrm{Al}_{x} \mathrm{Ga}_{(1-x)} \mathrm{As} / \mathrm{GaAs}(x=0.36)$ modulation-doped $p$-channel heterojunction sample ${ }^{6}$ with the [001] and [110] crystal axes along the longitudinal and transverse sources of $\mathbf{B}_{0}$, respectively. The following slowcooling procedure consistently yielded a strain-free sample. The probe with mounted sample was inserted into the roomtemperature cryostat, whose main chamber was then pumped and purged in a few cycles and left filled with $g$-He. The $l-\mathrm{N}_{2}$ jacket was then cooled for 20 min with the boiloff of a $l-\mathrm{N}_{2}$ tank, and subsequently filled with $l-\mathrm{N}_{2}$ over $20 \mathrm{~min}$. Cooling of the main cryostat chamber occurs slowly via the neck of the cryostat, lowering the temperature of sample and mounting grease to $77 \mathrm{~K}$ in $\sim 2 \mathrm{~h}$. Reduction to $4.2 \mathrm{~K}$ during the $l$-He fill occurred over $\sim 40 \mathrm{~min}$, while another $30 \mathrm{~min}$ of pumping on the main chamber achieved the $\lambda$-point $(\sim 2 \mathrm{~K})$. Strain-free results are evidenced by the ONMR spectra of Fig. 10(a). For contrast, a strained sample was obtained by lowering an identically mounted room-temperature sample into the cryostat at $4.2 \mathrm{~K}$ over $20 \mathrm{~min}$. The result is the quadrupolar triplet shown in the figure, whose origin in strain was confirmed by the fact that orientation dependence of the splitting is inconsistent with an electric field normal to the sample.

Dramatic resolution enhancement was obtained by using the CLSW-16 sequence during the $t_{1}$ evolution period. ${ }^{9,24}$ As shown in Fig. 10(b), this yielded a $4 \mathrm{~Hz}$ full width at half maximum (FWHM) spectrum, $10^{3}$-fold narrower than the $\sim 3.5 \mathrm{kHz}$ dipolar linewidth. [See spectra of Fig. 10(a).] This resolution allows atomically detailed POWER NMR images of single-electron densities and single-carrier electric-field distributions. ${ }^{5,8}$ Two additional details of the line narrowing are noteworthy. First, modifications to certain pulses in CLSW-16 were necessary to provide "second averaging" of the resulting average Hamiltonian, as described elsewhere. ${ }^{9,24,37,38}$ With this, linewidths to $<10 \mathrm{~Hz}$ were

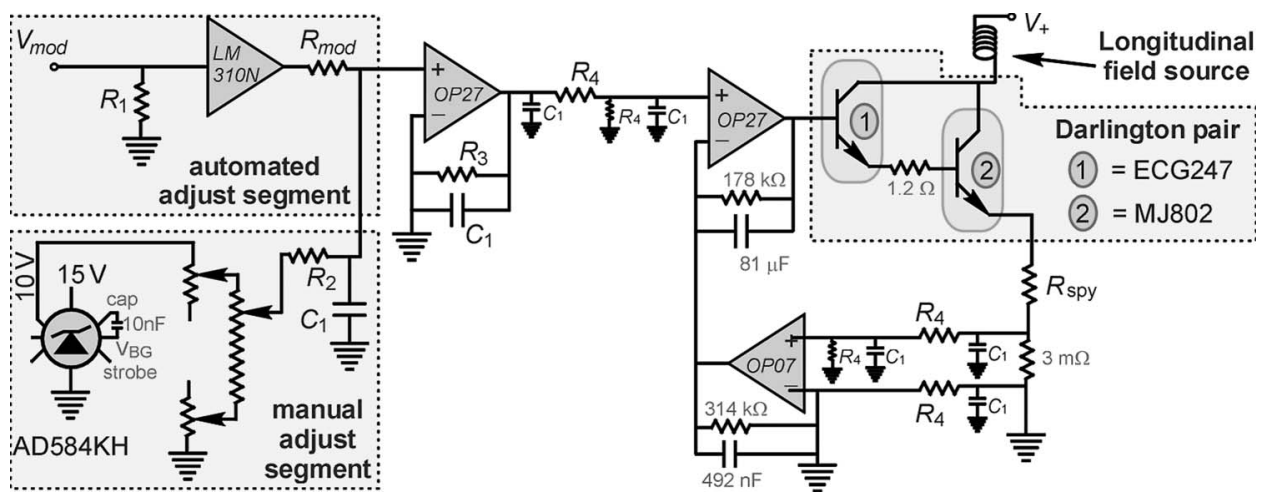

FIG. 9. Schematic of current-control circuit used to modulate the longitudinal magnetic field. Automated and manual controls are included. The resolution of automated control from $V_{\text {mod }}$ is set by $R_{\text {mod }}=33.1 \mathrm{k} \Omega$. Manual control is provided by variable resistors for course $(0-10 \mathrm{k} \Omega)$ and fine (two mechanically synchronized at $0-25 \Omega$ each) adjustments. These are zeroed during automated control. Current is regulated by high-power transistors [ECG247 (ECG Electronics), MJ802 (ON Semiconductor)] in a Darlington configuration, and may be monitored via the voltage across a spy resistor $\left(R_{\text {spy }}=33 m \Omega, \pm 5 \%\right.$, Kelvin KC500). Individual elements are a voltage follower (LM310, National Semiconductor), operational amplifiers (OP27, OP07, Analog Devices), a Zener diode (AD584KH, Analog Devices), $C_{1}=470 \mathrm{nF}$ and $\left(R_{1}, R_{2}, R_{3}\right.$, and $\left.R_{4}\right)=(2.05,9.98,9.93$, and $315 \mathrm{k} \Omega$ ). 

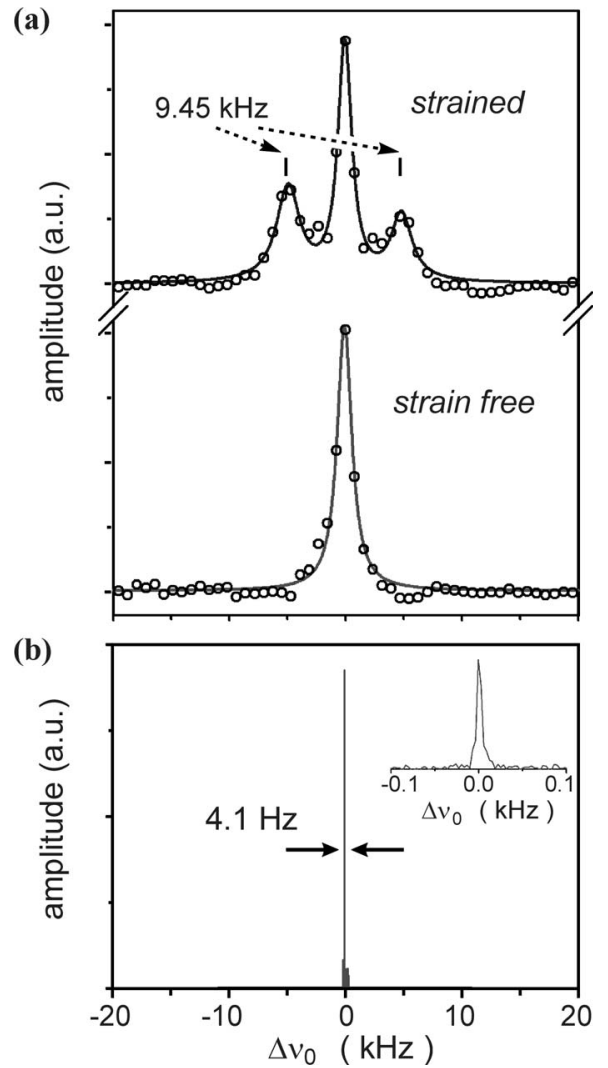

FIG. 10. ${ }^{71} \mathrm{Ga}$ LBD ONMR spectra of an AlGaAs/GaAs heterojunction at $2 \mathrm{~K}$ with $\mathbf{B}_{0}$ along the [001] crystal axis. (a) Spin-echo spectra that demonstrate our strain-free sample mounting procedure. (b) CLSW-16 spectra (4 Hz FWHM) for POWER NMR experiments. The inset shows an expanded view.

common, while no worse than $\sim 30 \mathrm{~Hz}$ FWHM was obtained in tens of experiments. Without the modification, we observed unwanted doublet structure arising from residual terms in the average Hamiltonian that were two to three times larger than the FWHM of a properly second-averaged line. The second noteworthy detail is that improved line narrowing occurred when using $\pi / 2$ pulse times at integer multiples of the rf cycle. This choice mitigates a breakdown of the rotating-wave approximation, allowing the pulse sequence to act as intended even though, at the low fields used in our experiments, $90^{\circ}$ pulses typically included only three to ten rf cycles.

Spin-echo-based LBD-detected NMR experiments were performed for initial characterization of the hyperfine shifts that result from optical excitation of spin-polarized electrons in the sample, apparatus, and conditions presented here. Figure 11(a) shows a combination rf and optical stimulated-echo pulse sequence for NMR evolution in $t_{1}$. The sequence only refocuses inhomogeneites due to terms in the nuclear spin Hamiltonian that are linear in $I_{z}$, leaving residual broadening from dipolar and quadrupolar spin interactions. Nonetheless, resolution is sufficient to reveal a hyperfine shift of $1.38 \pm 0.06 \mathrm{kHz}$ due to interactions with the excited electronic state. Furthermore, the linewidth of the central peak in the light-on spectrum is $1.78 \pm 0.05 \mathrm{kHz}$, about $450 \mathrm{~Hz}$ broader than in the light-off spectrum. This change reflects an inhomogeneous distribution of electron density (and thus, (a)

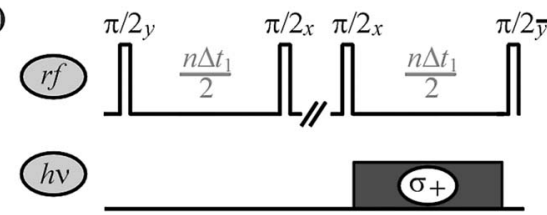

(b)

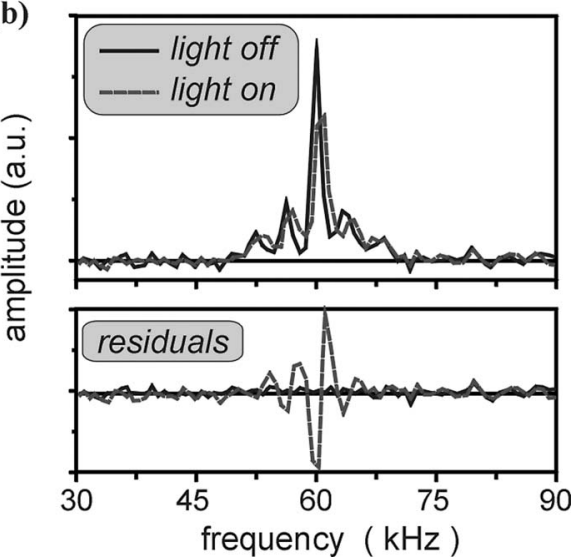

FIG. 11. Stimulated-echo sequence and spectra probing optically induced hyperfine shifts. (a) Combination rf/optical sequence applied during NMR evolution. The first $\pi / 2$ pulse is the preparation pulse of Fig. 8(a), while the second stores a component of magnetization from the first $t_{1} / 2=n \Delta t_{1} / 2$ period along $B_{0}$ in an essentially static state. The subsequent delay can accommodate arbitrary events and the following $\pi / 2$ stimulates a spin echo from stored magnetization after the second $t_{1} / 2$ period. Circularly polarized $\left(\sigma_{+}\right)$optical excitation of electron spins during the second $t_{1} / 2$ window yields net nuclear spin evolution due to the hyperfine interaction. (b) Stimulated-echo spectra obtained with and without the optical excitation noted in (a) and residuals of light-on minus light-off (dashed) and fitted light-off minus light-off spectrum (solid). Hyperfine interactions shift the light-on spectrum relative to light off by $0.69 \mathrm{kHz}$ (half the hyperfine shift as the light is on for $t_{1} / 2$ ). Both spectra were shifted from zero frequency using time-proportional phase incrementation (Ref. 39). The pattern of five peaks in each resulted from strain-induced quadrupole triplet [as in Fig. 10(a)] with additional peaks midway between central and satellite transitions due to pathways that mix the corresponding coherences during the delay period of the sequence in (a).

hyperfine shifts) among the nuclei contributing to the NMR spectrum. A similar hyperfine shift and altered line shape were observed previously in bulk GaAs at $77 \mathrm{~K}$ by using the time-sequenced optically detected NMR paradigm with steady state Hanle-effect optical detection. ${ }^{4}$ In contrast to that result and the stimulated echo of Fig. 11, the POWER NMR synchronization of Fig. 5 yields a pure hyperfine spectrum without dipolar or quadrupolar broadening. Such experiments provide $10^{3}$-fold resolution enhancement, as shown in Fig. 10(b). Analysis of such pure hyperfine spectra provides a detailed model for the wavefunction of quantumconfined electronic states that are responsible for the hyperfine interaction. ${ }^{9}$

In summary, we have detailed design specifications for a time-sequenced ONMR spectrometer with LBD detection. This includes (1) cryogenic systems and requirements for strain-free sample mounting, (2) a NMR probe with a nonpersistent superconducting magnet as the primary field source, multinuclear rf excitation coils, and a sampletemperature monitor, (3) optical systems for ONP and LBD, (4) the NMR spectrometer for observation of the LBD with high sensitivity in a narrow bandwidth, and (5) systems con- 
trol via PC communication to on-board cards for GPIB and coherent rf synthesis, and to a digital word generator for peripheral controls. Importantly, we demonstrated event synchronization among these elements as required for POWER NMR. This enables high-resolution measurement of small sample perturbations in spite of the orders-of-magnitude larger background interactions that otherwise hamper solidstate NMR. The high-resolution NMR results $(4.1 \mathrm{~Hz}$ FWHM) presented here are without precedent in the ONMR literature and are at the state-of-the-art for any solid-state NMR method. Recently, this enabled our atomic-scale imaging of quantum-confined electron-hole pairs (excitons) that resided in the tail of the interfacial electric field of the sample used here. ${ }^{9}$ Similar applications to image nanoscale electronic properties in quantum wells, dots, or any variety of structured semiconductor material can provide vital understanding of function and suggest guidelines to tune device performance.

\section{ACKNOWLEDGMENTS}

We thank A. Ksendov, J. Liu, and F. Grunthaner of the NASA Jet Propulsion Laboratory Center for Space Microelectronics for providing the sample and preliminary PL characterization, and L. Burnett and A. Perry for cryogenics advice. This work was supported by the NSF Program in Materials Synthesis and Processing (Grant No. CHE9612226) and by NASA through the Caltech President's Fund.

${ }^{1}$ G. Lampel, Phys. Rev. Lett. 20, 491 (1968).

${ }^{2}$ D. Paget, G. Lampel, B. Sapoval, and V. I. Safarov, Phys. Rev. B 15, 5780 (1977).

${ }^{3}$ A. I. Ekimov and V. I. Safarov, JETP Lett. 15, 319 (1972).

${ }^{4}$ S. K. Buratto, D. N. Shykind, and D. P. Weitekamp, Phys. Rev. B 44, 9035 (1991).

${ }^{5}$ S. K. Buratto, D. N. Shykind, and D. P. Weitekamp, J. Vac. Sci. Technol. B 10, 1740 (1992).

${ }^{6}$ J. A. Marohn, P. J. Carson, J. Y. Hwang, M. A. Miller, D. A. Shykind, and D. P. Weitekamp, Phys. Rev. Lett. 75, 1364 (1995).

${ }^{7}$ D. Gammon, S. W. Brown, E. S. Snow, T. A. Kennedy, D. S. Katzer, and D. Park, Science 277, 85 (1997).
${ }^{8}$ J. G. Kempf and D. P. Weitekamp, J. Vac. Sci. Technol. B 18, 2255 (2000).

${ }^{9}$ J. G. Kempf, M. A. Miller, and D. P. Weitekamp (unpublished).

${ }^{10}$ P. Khandelwal, A. E. Dementyev, N. N. Kuzma, S. E. Barrett, L. N. Pfeiffer, and K. W. West, Phys. Rev. Lett. 86, 5353 (2001).

${ }^{11}$ M. Eickhoff, B. Lenzmann, D. Suter, S. E. Hayes, and A. D. Wieck, Phys. Rev. B 67, 085308 (2003).

${ }^{12}$ D. Paget, Phys. Rev. B 25, 4444 (1982).

${ }^{13}$ M. Eickhoff and D. Suter, J. Magn. Reson. 166, 69 (2004).

${ }^{14}$ J. M. Kikkawa and D. D. Awschalom, Science 287, 473 (2000).

${ }^{15}$ A. Goto, S. Ohki, K. Hashi, and T. Shimizu, Rev. Sci. Instrum. 77, 093904 (2006).

${ }^{16}$ M. Henini, Nanoscale Res. Lett. 1, 32 (2006).

${ }^{17}$ G. D. Scholes and G. Rumbles, Nat. Mater. 5, 683 (2006).

${ }^{18}$ S. W. Koch, M. Kira, G. Khitrova, and H. M. Gibbs, Nat. Mater. 5, 523 (2006).

${ }^{19}$ S. A. Wolf, D. D. Awschalom, R. A. Buhrman, J. M. Daughton, S. von Molnar, M. L. Roukes, A. Y. Chtchelkanova, and D. M. Treger, Science 294, 1488 (2001).

${ }^{20}$ A. Kent, Experimental Low-Temperature Physics (AIP, New York, 1993).

${ }^{21}$ S. S. Andrews and S. G. Boxer, Rev. Sci. Instrum. 71, 3567 (2000).

${ }^{22}$ J. Y. Hwang, Ph.D. thesis, Caltech, 1994.

${ }^{23}$ J. A. Marohn, Ph.D. thesis, Caltech, 1996.

${ }^{24}$ H. M. Cho, C. J. Lee, D. N. Shykind et al., Phys. Rev. Lett. 55, 1923 (1985)

${ }^{25}$ W. Hanle, Z. Phys. 30, 93 (1924).

${ }^{26}$ M. I. Dyakonov and V. I. Perel, in Optical Orientation, edited by F. Meier and B. P. Zakharchenya (North-Holland, Amsterdam, 1984), Vol. 8, p. 11.

${ }^{27}$ V. L. Berkovits, A. I. Ekimov, and V. I. Safarov, Sov. Phys. JETP 38, 169 (1974).

${ }^{28}$ M. I. D'yakonov and V. I. Perel', Sov. Phys. JETP 38, 177 (1974).

${ }^{29}$ N. Bloembergen, in Proceedings of the 11th Colloque Ampere Conference on Electric and Magnetic Resonance, edited by J. Smidt (North-Holland, Amsterdam, Eindhoven, 1962), p. 225.

${ }^{30}$ E. Brun, R. Hahn, W. Pierce, and W. H. Tantilla, Phys. Rev. Lett. 8, 365 (1962).

${ }^{31}$ D. Gill and N. Bloembergen, Phys. Rev. 129, 2398 (1963).

${ }^{32}$ K. A. Dumas, J. F. Soest, A. Sher, and E. M. Swiggard, Phys. Rev. B 20 , 4406 (1979).

${ }^{33}$ T. Sleator, E. L. Hahn, M. B. Heaney, C. Hilbert, and J. Clarke, Phys. Rev. Lett. 57, 2756 (1986).

${ }^{34}$ R. K. Sundfors, Phys. Rev. B 10, 4244 (1974).

${ }^{35}$ G. P. Flinn, R. T. Harley, M. J. Snelling, A. C. Tropper, and T. M. Kerr, Semicond. Sci. Technol. 5, 533 (1990).

${ }^{36}$ A. Abragam, Principles of Nuclear Magnetism (Clarendon, Oxford, 1961).

${ }^{37}$ A. Pines and J. S. Waugh, J. Magn. Reson. (1969-1992) 8, 354 (1972).

${ }^{38}$ J. G. Kempf, Ph.D. thesis, Caltech, 2000.

${ }^{39}$ G. Drobny, A. Pines, S. Sinton, D. P. Weitekamp, and D. Wemmer, Faraday Symp. Chem. Soc. 13, 49 (1979). 